\title{
Study on Corner Reflectors Identification in Highway Deformation Monitoring
}

\author{
Xue Min Xing ${ }^{1,2,3}$, De Bao Wen ${ }^{2}$ and Fang Bin Zhou ${ }^{2}$ \\ ${ }^{1}$ Key Laboratory of Special Environment Road Engineering of Hunan Province, \\ Changsha University of Science \& Technology, Changsha, 410004, China; \\ ${ }^{2}$ Changsha University of Science \& Technology, Changsha, 410004, China; \\ ${ }^{3}$ School of Info-Physics and Surveying Engineering, Central South University, \\ Changsha 410083, China \\ 28008642@qq.com,xing28008642@Gmail.com,38896263@qq.com
}

\section{Abstract}

CRInSAR is a newly developed technique to monitor ground deformation. In CRInSAR algorithm, the identification of Corner Reflectors in SAR images is necessary. Due to the uncertainty of traditional identification method, a new method based on the intensity and correlation coefficient of each CR candidates in SAR images is presented. The method has been successfully used to determine the locations of 11 CRs installed along a highway in six SAR images over the study area. The results show that the identification accuracy of the new method is about 1 pixel. It is effective and reliable especially in the area with lots of lightspots around the CR points. The method proposed can play important role in the highway deformation monitoring within CRInSAR algorithm.

Keywords: CRInSAR, Corner Reflector, $S A R$ Coordinates, Highway, Deformation Monitoring

\section{Introduction}

CRInSAR (Conen Reflector Interferometric Synthetic Aperture Radar) is a newly developed technique of DInSAR (Differential InSAR). It can help us to obtain the deformation information based on the analysis of the phase changes of groups of CR (Corner Reflector) points instanled in the study area preliminarily [1]. For that the backscatter characteristics of these C $\mathrm{R}$ points perform highly better than that of surroundings, each of them appears to be single light spot in SAR images (See Figure 1). CRInSAR has been successfully applied in ground deformation monitoring [2-3]. It can be obviously seen that due to the special structure and installation, the CR points can be identified easily from the image. In addition, with CRInSAR algorithm, the geocoding error in the processing of DInSAR caused by the external DEM can be avoided. As discussed above, it can be inferred that us ng CR points has great advantages in the application of deformation monitoring [4].

In the deformation monitoring algorithm using CRInSAR, the identification of CR points is a necessary step which can help us to determine the exact location (precise row and column information) of each CR point in each SAR image [5]. The traditional method used the longitude and latitude information of each CR point to inverse the initial row and column coordinate based on the Range and Doppler (RD) model and finally inversed the location of CR point under visual check and comparison[6]. This method has the following limitations: (1) there exist deviation between the inversed location and the real location of CR point; (2) due to the environment of the study area, there may appear to be more than one light spot 
which may confuse the visual check and as a result lead the final location of the CR point hard to determine; (3) great uncertainty exists during the process of personal visual check and comparison. Due to these limitations, we will attempt to propose a more effective algorithm called Double Thresholding Algorithm (DTA) to determine the locations of CR points. This method will be applied to identify three groups of CR points installed along the highway in China from six PALSAR images.

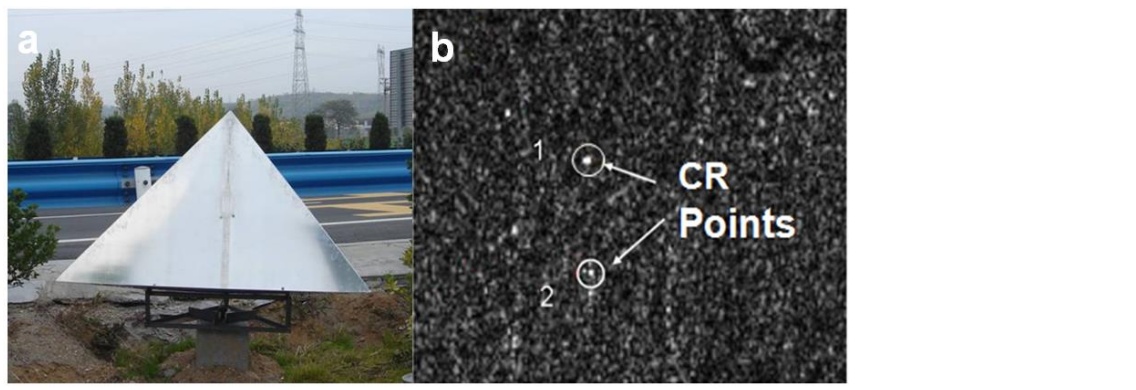

Figure 1. Photo of Artificial CR (a) and its Corresponding Performance in SAR Image (b)

\section{Double Thresholding Algorithm (DTA) to Identify the CR Points}

\subsection{Principles}

The inversion processing of the CR points' coordinates based on RD model has large system errors and may be influenced easily by the environment which may lead the phenomenon that more than one light spots appeay around the CR point. As shown in Figure $2 \mathrm{a}$, it can be seen that too many light spots-distributed in the area which make it difficult to identify the true CR points. Due to this, the Double Thresholding Algorithm (DTA) will be proposed in this paper. Because of the high reflectivity, the CR points perform to have high intensity and corresponding high conerence. Considering both these two factors, we firstly use the intensity index to choose goup of points as candidates initially, and then use the correlation coefficient index as criterion to determine the final location from the candidates. Setting the intensity value of the ${ }^{k}$-th CR candidate to be expressed as ${ }^{d B_{k}}$ which can be extracted from the intensity image, the corresponding correlation coefficient ${ }^{\gamma_{k}}$ can be calculated by the mean of time series coherence, which can be written as[7]:

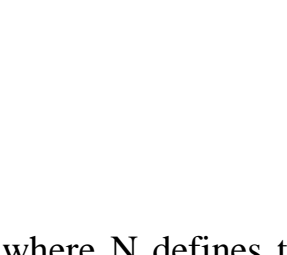

$$
\gamma_{k}=\frac{\sum_{p=1}^{N} \gamma_{p}}{N}
$$

where $\mathrm{N}$ defines the index of the time series interferometric pair; ${ }^{\gamma_{p}}$ defines the coherence value of this CR candidate in the ${ }^{p}$-th interferometric pair $[8,9]$. 

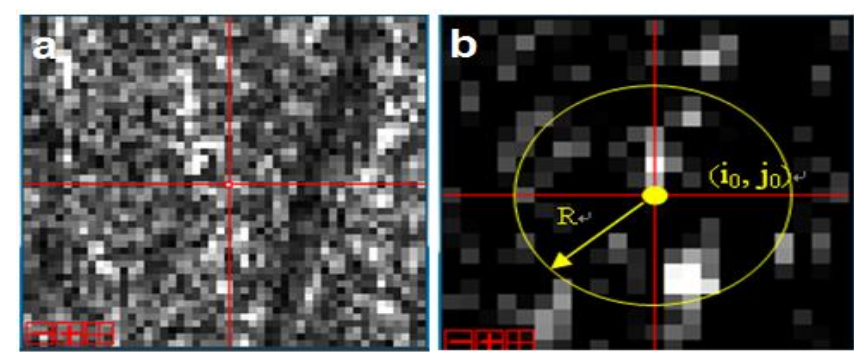

Figure 2. Possible Distribution of Light Spots (a) and Position of the CR

Candidates (b)

\subsection{Operating Process of the Algorithm}

According to the principles discussed above, supposing the number of CR point needed to be identified in one image is $\mathrm{M}+1$, the flow step of DTA is summarized below (the corresponding algorithm flow is shown in Figure 3):

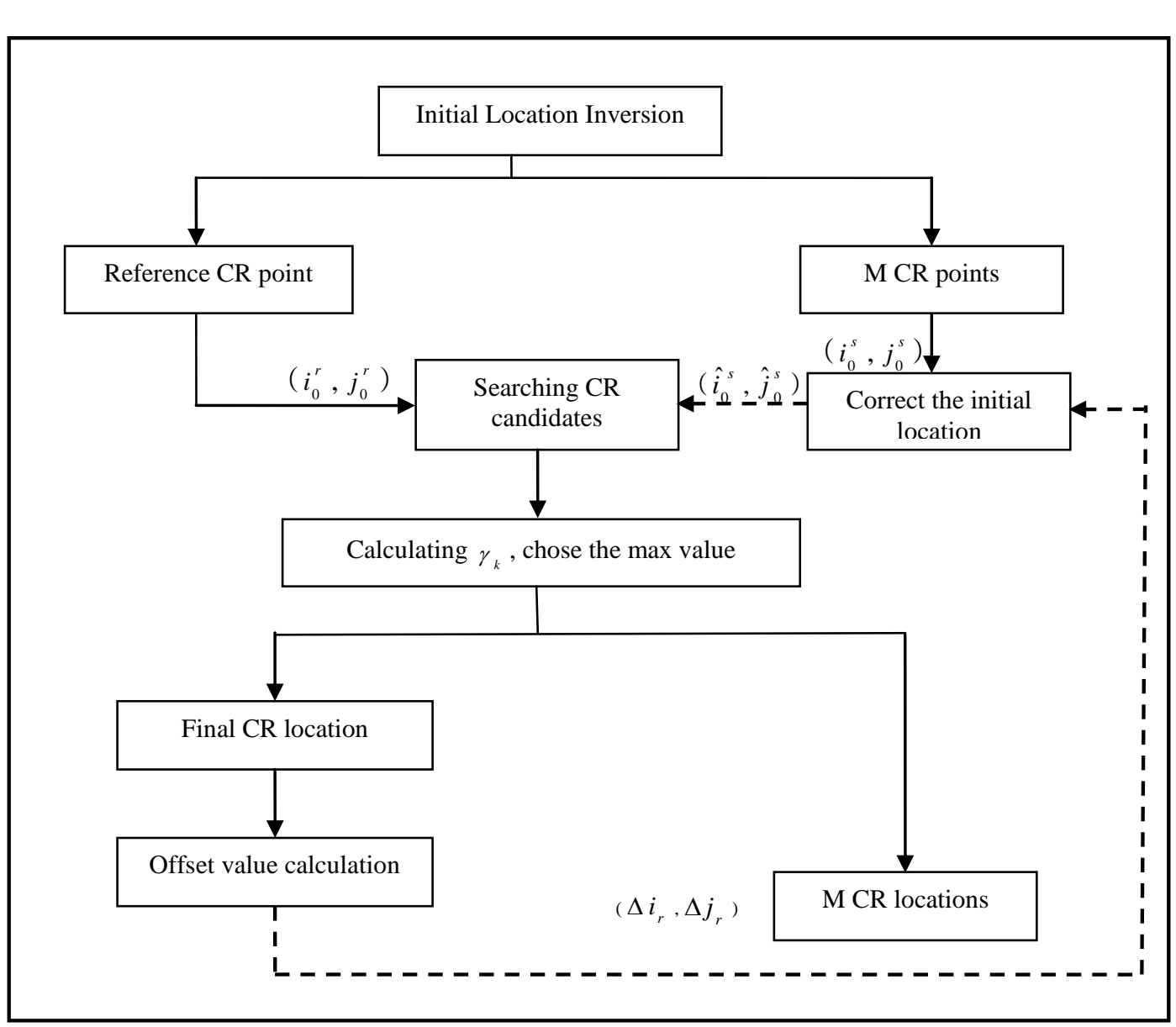

Figure 3. Algorithm Flow of the DTA

(1) Calculating the initial row and column information using RD model according to the altitude and latitude value obtained from the GPS receiver. The detail operating process of $\mathrm{RD}$ model inversion is introduced in [6]. One of the $\mathrm{M}+1 \mathrm{CR}$ points is chosen as reference 
point, the initial row and column value worked out is written as $\left({ }^{i^{r}}, j_{0}{ }^{r}\right)$, while that of the rest $M$ CR points is written as $\left({ }^{i_{0}^{s}}, j_{0}^{s}\right)$, where ${ }^{s=1, \cdots, M}$;

(2) Searching each of the light spots according to the intensity value in the area with the location $\left({ }^{i_{0}{ }^{r}}, j_{0}{ }^{r}\right)$ as center, $R$ as radius(shown in Figure $2 \mathrm{~b}$ ). The points with intensity value above the threshold index are chosen as candidates, being written as $\left({ }^{i_{r}}{ }^{1},{ }^{j_{r}}{ }^{1}\right),($ $\left.i_{r}{ }^{2}, j_{r}{ }^{2}\right), \ldots,\left({ }^{i_{r}}{ }^{k}, j_{r}{ }^{k}\right)$. As discussed above, due to the limitation of the natural environment, there will be the phenomenon that the contrast between the reflections of CR points and natural points is not obviously enough. We can use the ENVI software to enhanêe the contrast in order to make the identification of CR points more easily ;

(3) Calculating the value of ${ }^{\gamma_{k}}$ on each CR candidates according to Eq, (The row and column value of the ${ }^{k}$-th CR candidate with max $\gamma_{k}$ can be written as $\left(i^{k}, j\right.$, , which can be taken as the final row and column value of this reference CR point ( $j$ ):

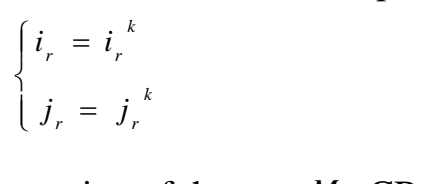

(4) Correcting the initial location information of the rest $M$ CR points. For the error of the initial row and column value obtained from RD model has systematic characteristic, the total offset value of all the calculated locations reference to the true ones are identical. According to this, after calculating the reference $\mathrm{CR}$ pointes row and column information $\left({ }^{i_{r}}, j_{r}\right)$, the offset value reference to he initial row and column value $\left(i_{0}{ }^{r}, j_{0}{ }^{r}\right.$ ) can be written as $\left(\Delta i_{r}, \Delta j_{r}\right)$ :

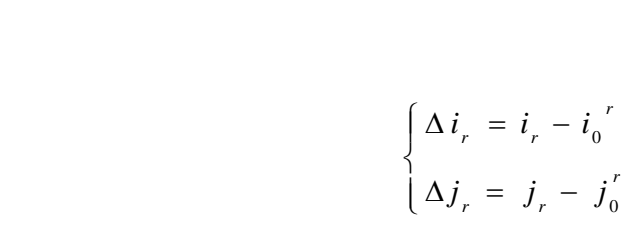

The initial row and column value after correcting can be written as $\left(\hat{i}_{0}^{s}, \hat{j}_{0}^{s}\right)$, which can be calculated as follows.

where $s=1, \cdots, M$, then the $\left(\hat{i}_{0}^{s}, \hat{j}_{0}^{s}\right)$ can be taken as center point, with step (2)- (3) being $\left\{\begin{array}{l}\hat{i}_{0}^{s}=i_{0}^{s}+\Delta i_{r} \\ \hat{j}_{0}^{s}=j_{0}^{s}+\Delta j_{r}\end{array}\right.$
taken as center point, with step $(2)$

\section{Experiment}

\subsection{The Installation and Design of $\mathrm{CR}$}

In order to validate the proposed DTA above, three groups of CR points are installed along a highway in Henan province of China. Figure 4 shows the distribution of the CR points in 
the master intensity image. The goal to install the CR points along the highway is to help monitoring the ground deformation of the highway. CR01, CR05 and CR12 are chosen as reference points in each group respectively.

In order to determine the locations of CR in SAR Images immediately and accurately, the shape and installation should follow certain discipline. The trihedral solid aluminium sheets are used in the design, which indicates that the scattering surface is isosceles right triangle (see Figure 5), the corresponding max RCS (Radar Cross Section Area) can be expressed as [10]:

$$
R C S_{\max }=\frac{4 \pi l^{4}}{3 \lambda^{2}}
$$

where ${ }^{l}$ defines the length of corner reflector being $1.2 \mathrm{~m}$ in the experiment. Puring the installation of the CR points, the following conditions are considered [11-12]

(1) Azimuth: the orientation of CR should be coincide with that of rada LOS (light of sight), so that the RCS can reach the max value. The orientation is correlated with the orbit angle of radar, the longitude of CR's location and the azimuth of CR's hemline. The corresponding relationship function can be written as

$$
\beta=\arcsin \frac{ \pm \cos \alpha}{\cos \zeta}
$$

where ${ }^{\beta}$ defines the azimuth of CR's hemline; ${ }^{\alpha}$ defines the slope angle of Radar orbit; $\zeta$ defines the longitude of CR's location;

(2) Longitudinal attitude: The horizontal side should be keep level before the longitudinal attitude being determined. The longitudinal attitude should be adjusted according to Figure 5 .

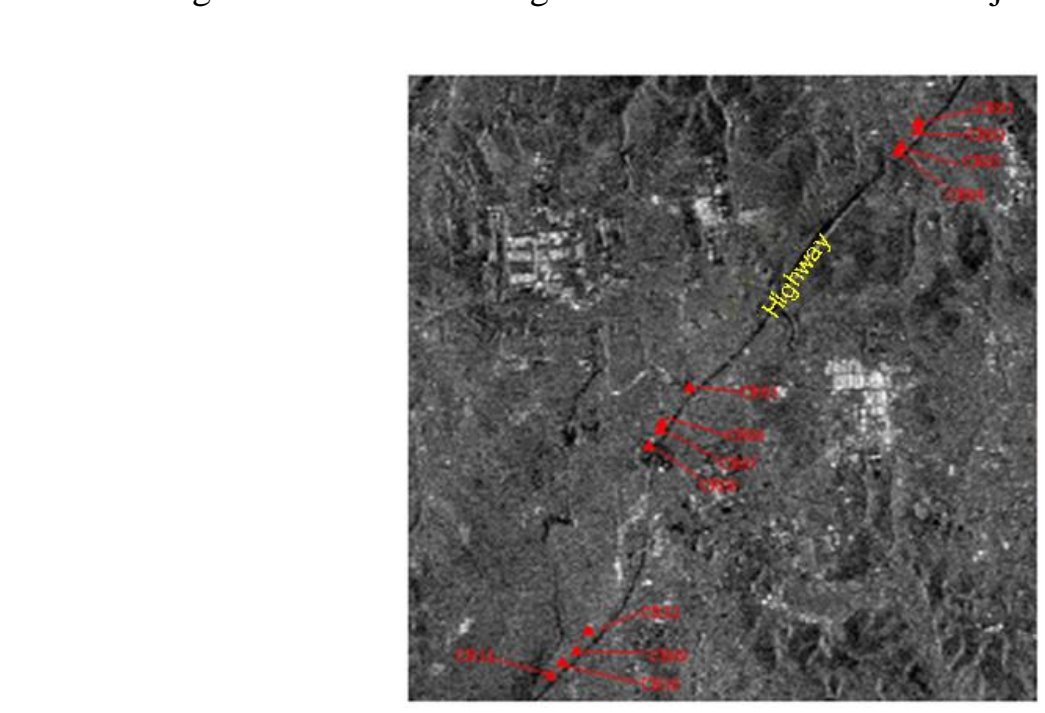

Figure 4. The Locations of CR Points along the Highway in the Master Image 

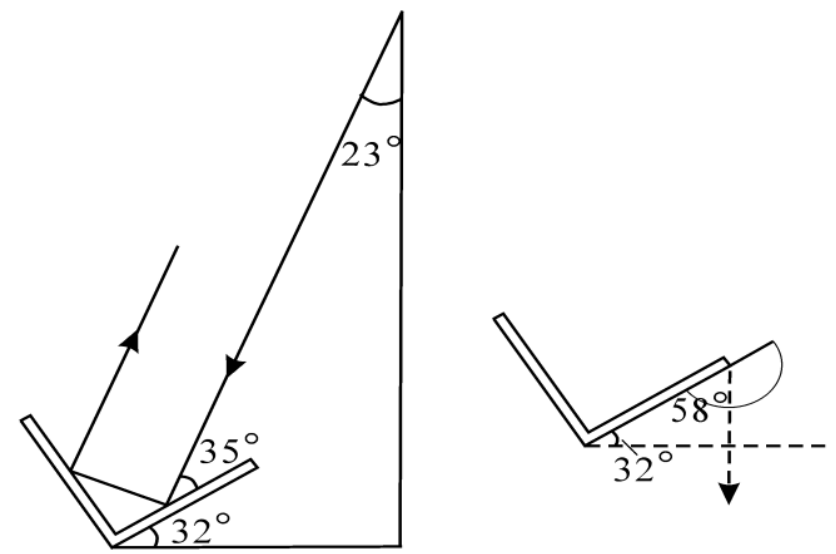

Figure 5. Isosceles Right Triangle Trihedral CR and Adjustment of CR's Longitudinal Attitude

\subsection{Identification of CR Points}

The SAR images used in the experiment are six PALSAR images acquired from December 2008 to December 2009. There are many houses and metal fences in the study area which have high reflectivity and perform to be lots of light spots in the image near the CR points. As a result the method using only RD model and artificial comparison can not satisfy the requirement of high identification accuracy As discussed above, the DTA proposed here is used to identify the CR points in the sir mages respectively. In the experiment $R$ is set to be 10 pixels while the threshold value of intensity being $1.0 \mathrm{~dB}$. The algorithm is an iterative process and finally the location with the max alue of ${ }^{\gamma_{k}}$ is determined to be the final CR point.

With use of the method discussed, the 1 CR points' final locations are determined in the six images, respectively. The final performance of the detected CR points is shown in Figure 6. It can be obviously seen that the $\mathrm{CR}$ points detected are much brighter than the environment. With CR10 being destroyed accidentally during the highway construction, 11 CR's accurate roy and columinformation are determined. Table 1 lists the exact row and column information determined with RD model inversion and DTA. It can be seen that DTA corrected and improved the column information of each CR point with max value of 4 pixels at CR 1 and min valae of 1 pixel at CR 3 .

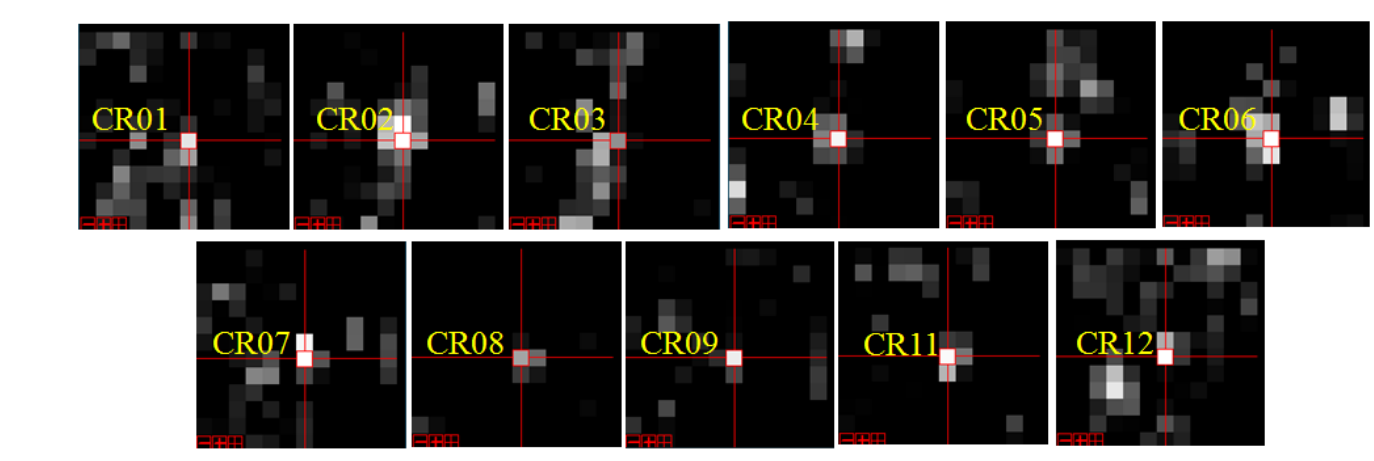

Figure 6. Detail of CR Points in Master Image 


\section{Table 1. SAR Coordinates of CR Points in Master Image Determined with RD Inversion and DTA}

\begin{tabular}{ccccc}
\hline \multirow{2}{*}{$\begin{array}{c}\text { Index of } \\
\text { CR }\end{array}$} & row & column & row & column \\
\hline cr01 & 3840 & 5160 & 3840 & 5156 \\
cr02 & 3868 & 5160 & 3868 & 5157 \\
cr03 & 3913 & 5112 & 3913 & 5111 \\
cr04 & 3930 & 5100 & 3930 & 5096 \\
cr05 & 4650 & 4468 & 4650 & 4465 \\
cr06 & 4763 & 4384 & 4763 & 4380 \\
cr07 & 4782 & 4376 & 4782 & 4373 \\
cr08 & 4832 & 4340 & 4832 & 4338 \\
cr09 & 5455 & 4120 & 5455 & 4117 \\
cr11 & 5532 & 4040 & 5532 & 4038 \\
cr12 & 5398 & 4152 & 5398 & 4150 \\
\hline
\end{tabular}

\subsection{Validation}

The reference distances between each two specified CR points in different SAR images need to be accordant. Due to this, we calculated the reference distances between each neighbored two of the $11 \mathrm{CRs}$ in all the six images. In order to test if the reference distances in each slave image is consistent with that of the master image, we subtracted the reference distances in the master image from each of the five slave images and compared them in order to validate the identification accuracy of DTA. The comparison results including distances differences along both attitude and range direction is shown in Figure 7. From the comparison results we find that all the reference distances differences varied below $4 \mathrm{~m}$. According to the resolution of the six SAR-images the reference distances differences are all less than 1 pixel except the distance between CR07 and CR08 which is a little more than 1 pixel. This confirms tha the accuracy of DTA identification is about 1 pixel. It proved that the CR locations determined with the new method is accurate and reliable.
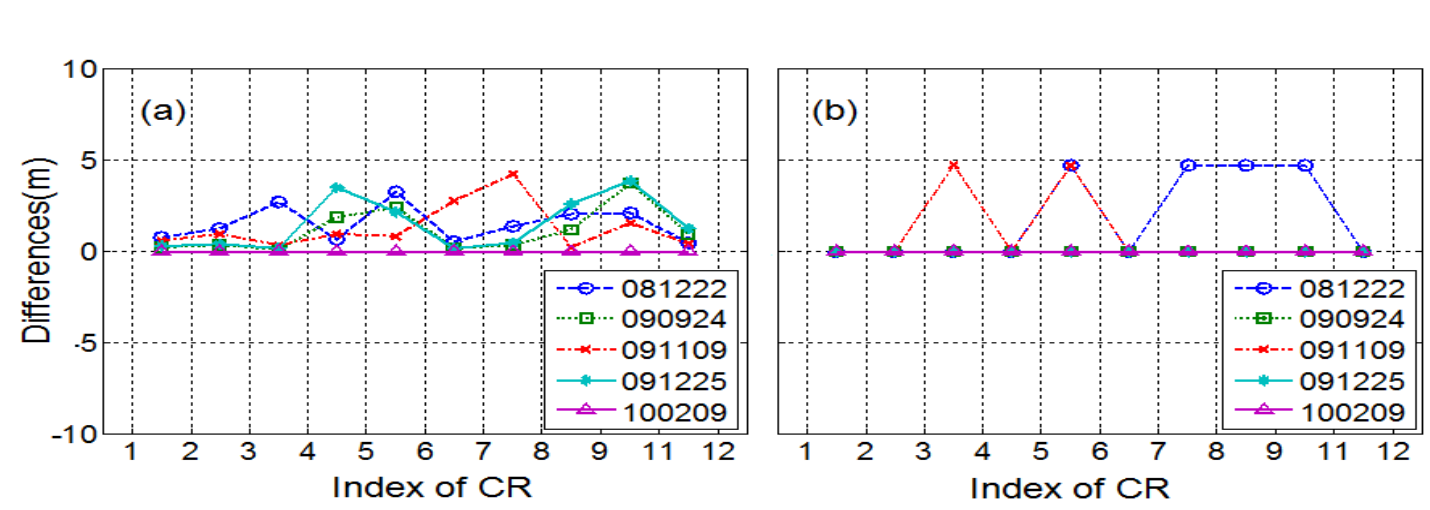

Figure 7. Differences of each Neighbored CRs' Distance in Five Slave Images Reference to that in the Master Image (a: Along the Attitude Direction, b: Along the Range Direction) 


\section{Conclusions}

In this paper, a new method to identify the CR points is proposed, which uses Double Thresholding Algorithm (DTA) taking the intensity threshold value as initial criterion while the correlation coefficient as the final criterion. The method is applied successfully to identify the $11 \mathrm{CR}$ points' locations installed along a highway and obtain their row and column information. The results show that the method proposed can be applied in the area where lots of lightspots exist near the CR points due to the severe environment. In this condition the traditional method based on RD model and visual check may lose effectiveness. The new method will not be limited to the circumstance and can avoid the artificial error during the personal visual check and comparison.

In order to validate the algorithm proposed, comparison of reference distances between each two specified CR points in different SAR images has been carried out. The comparison results show that the identification accuracy is about 1 pixel, so it can be concluded that the method presented in the paper is accurate and reliable. It can be applied to assist the CRInSAR algorithm in the ground deformation monitoring of highway. The future study will be focused on the application of DTA with CRInSAR algorithin to inverse the time series ground deformation of highway in Hunan province.

\section{Acknowledgements}

Project supported in part by National Nature Science Fơundation of China(No:41174001), Hunan Provincial Natural Science Foundation for Distinguished Young Scientists (Grant No. 14JJ1021), the key project of Hunan EduCation Department (Grant No.12A0020), Open Fund of Key Laboratory of Special Environment Road Engineering of Hunan Province (No: kfj130405).

\section{References}

[1] W. Fu, H. D. Guo and Q. Tian, "Landslide monitoring by corner reflectors differential interferometry SAR", International Joumal of Remote Sensing, vol. 31, (2010), pp. 6387-6400.

[2] B. Yu, G. X. Liu and Z. L. Li, "Subsidence detection by terraSAR-X interferometry on a network of natural persistent scatterers and artificial comer reflectors", Computers \& Geosciences, vol. 58, (2013), pp. 126-136.

[3] C. Y. Zhao, Z. Lu and Q. Zhrang, "Large-area landslide detection and monitoring with ALOS/ PALSAR imagery data over Northern California and Southern Oregon", USA, Remote Sensing of Environment, vol. 124, (2012), pp. 348-359.

[4] Y. Xia, H. Kaufmann and X. Guo, "Landslide Monitoring in The Three Gorges Area Using D-InSAR and Corner Reflectors" Photogrammetric Engineering and Remote Sensing, vol. 70, (2004), pp. 1167-1172.

[5] Y. Xia, "CR-Based SAR-Interferometry for Landslide Monitoring", in: IEEE 2008 International Geoscience and Remote Sensing Symposium, Boston, (2008), pp. 1-12.

[6] J. P. Zhou L. L. Tang and C. R. Li, "Development of Two Practical R-D Location Model and Precision Comparison between Them", Journal of Remote Sensing, vol. 5, (2001), pp. 191-197.

[7] Q. Chen. 9. X. Liu and Y. S. Li, "Automated Detection of Permanent Scatterers in Radar Interferometry", Algorithm and Testing Results, Acta Geodatica et Cartographica Sinica, vol. 35, (2006), pp. 112-117.

[8. R. Burgmann, G. E. Hilley and A. Ferretti, "Resolving vertical tectonics in the San Francisco Bay area from permanent scatter InSAR and GPS analysis", Geology, vol. 34, (2006), pp. 221-224.

[9] A. Ferretti, G. Savio and A Barzagi, "Submillimeter Accuracy of InSAR Time Series: Experimental Validation", IEEE Transactions on Geoscience and Remote Sensing, vol. 45, (2007), pp. 1142-1153.

[10] R. Xiang, J. P. Long and G. C. Feng, "Modeling atmospheric effects of corner reflector InSAR based on CGPS observations and meteorological data: A case study in Hong Kong", Engineering of Surveying and Mapping, vol. 40, (2008), pp. 47-49.

[11] T. Cheng and X. J. Shan, "Research on algorithm of CRInSAR and PSInSAR Combined Calculation", Earthquakes, vol. 27, (2007), pp. 64-71.

[12] J. Hu, J. J. Zhu and Z. W. Li, "On CR-InSAR and Its Morbid Equation”, Journal of Geometics, vol. 32, (2007), pp. 30-32. 


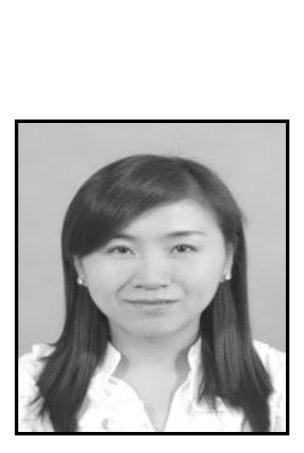

\section{Author}

Xuemin Xing, she was born in 1983, Liaoning province in China. She acquired the doctor degree in Geomatrics and Surveying, Central South University, Changsha, China in 2011. The major field is the application of PS-DInSAR in deformation monitoring, combined CRInSAR and PSInSAR, ground deformation monitoring in mining area using time series InSAR technique.

She is a teacher in School of Traffic and Transportation Engineering, Changsha University of Science \& Technology. She has published couple of papers in Chinese Journal of Geophysics, ICIC Express Letters and Chinese Journal of Central South University, such as article entitled "Subsidence Monitoring in Mining Area Based on PSInSAR and "Linear Subsidence Monitoring in mining area using Constrained PSInSAR" indexed by EI and articles entitled "Time Series Ground Subsidence Inversion in Mining Area based on CRInS AR and PSInSAR Integration" and "Study on Detecting the Regional Linear Subsidence Based on CRInSAR and PSInSAR Integration" indexed by SCI.

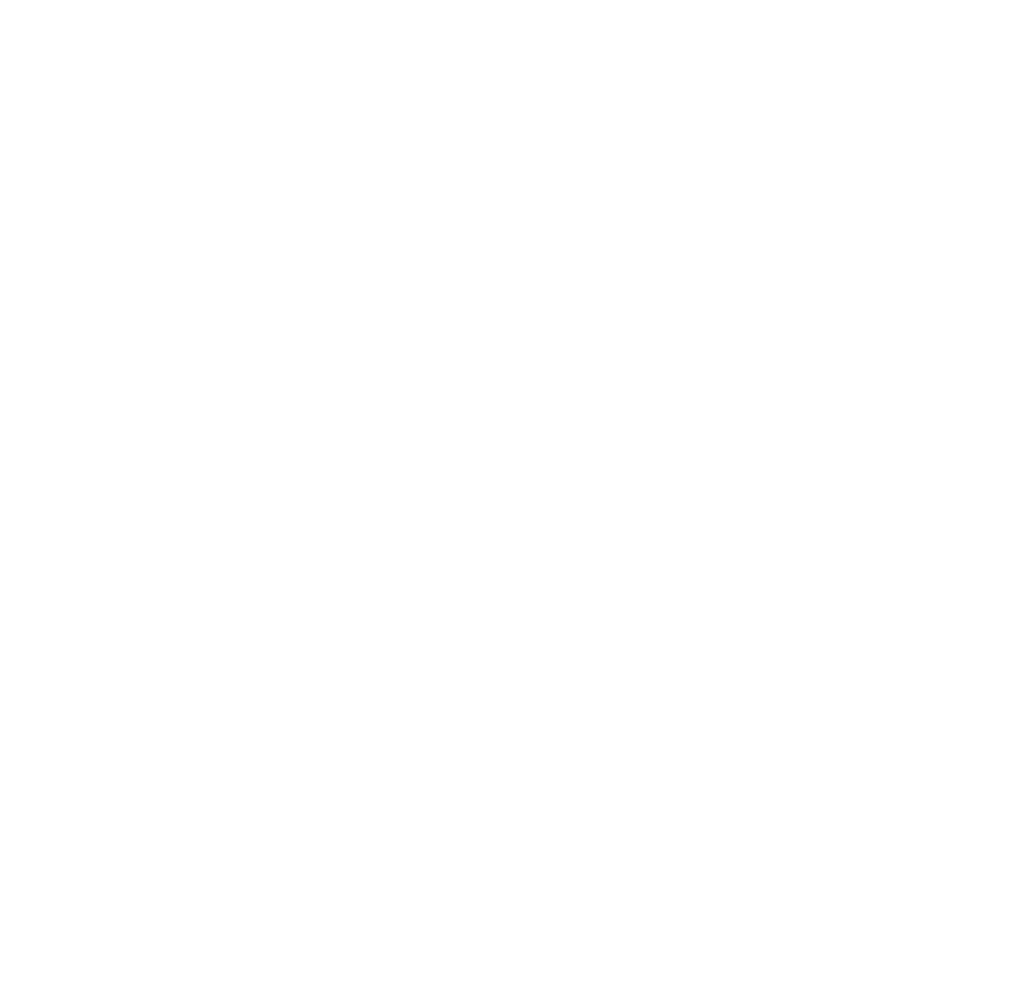


International Journal of Multimedia and Ubiquitous Engineering Vol. 9, No. 12 (2014)

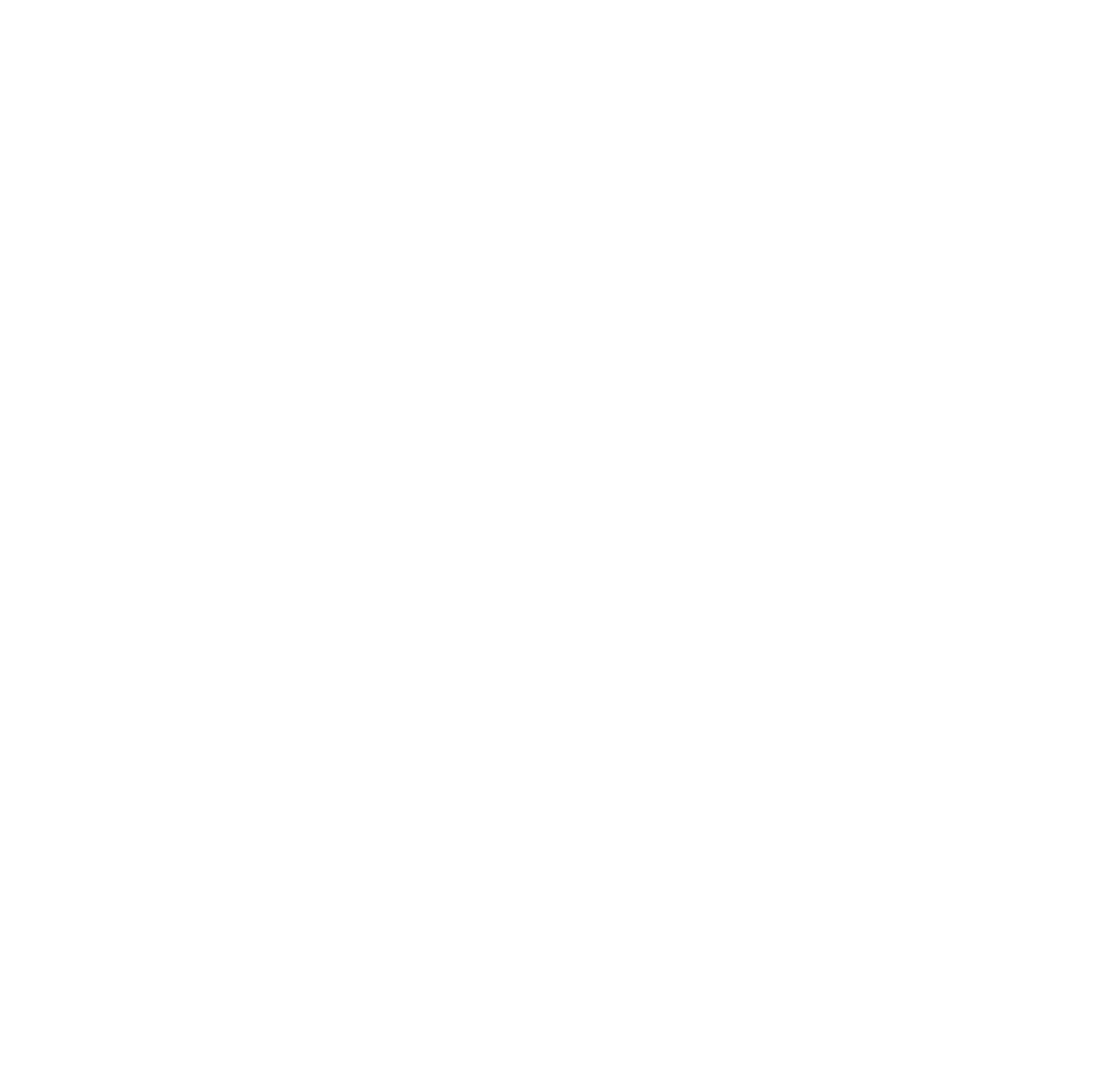

